\title{
Extraordinary optical gain from silicon implanted with erbium
}

\author{
M. A. Lourenço, ${ }^{\text {a) }}$ R. M. Gwilliam, and K. P. Homewood ${ }^{\text {b) }}$ \\ Advanced Technology Institute, Faculty of Engineering and Physical Sciences, University of Surrey, \\ Guildford, Surrey GU2 7XH, United Kingdom
}

(Received 27 June 2007; accepted 19 September 2007; published online 5 October 2007)

\begin{abstract}
Here we report on measurements of optical gain at $1.5 \mu \mathrm{m}$ in crystalline silicon. Gain is achieved by the incorporation of the rare earth erbium in silicon. A method was developed to enable the gain measurement in short silicon waveguides. Crucially, gain values obtained are significantly greater than previously supposed. We have measured a lower limit for the optical cross section for $\mathrm{Er}^{3+}$ of $5 \times 10^{-19} \mathrm{~cm}^{2}, 30$ times higher than previously anticipated. Given these higher values, this system now offers a realistic route to the production of electrically pumped silicon optical amplifier and laser devices using standard silicon process technology. (c) 2007 American Institute of Physics.
\end{abstract} [DOI: $10.1063 / 1.2797975$ ]

Recent years have seen tremendous advances in Si photonic devices. ${ }^{1-6}$ The last hurdles to implementation of full $\mathrm{Si}$ photonic systems remain the electrically pumped optical amplifiers and lasers in Si using complementary metal-oxide semiconductor (CMOS) compatible technology. The preferred wavelength for such systems is $1.5 \mu \mathrm{m}$. Optical gain in $\mathrm{Si}$ has been shown recently utilizing the Raman effect ${ }^{2-4}$ and phase matched four wave mixing, ${ }^{5}$ although neither is capable of being electrically pumped. One report shows optically pumped lasing in crystalline $\mathrm{Si}^{6}{ }^{6}$ at $1.27 \mu \mathrm{m}$, attributed to the so called $A$ center introduced using surface texturing. It is unclear whether this center, whose nature and origin is unknown, can be incorporated using CMOS processes or electrically pumped. The gain curve of this center is too narrow, $\sim 1 \mathrm{~nm}$, for optical amplifiers, where a broad gain spectrum is needed. ${ }^{5}$ Here we show gain over $150 \mathrm{~nm}$, centered at $1550 \mathrm{~nm}$, ideally suited for dense wavelength division multiplexing.

Optical gain in semiconductors is almost universally achieved by population inversion of carriers in a $p-n$ junction. This requires high gain as the high doping required leads to high free carrier and Auger losses, and is only achievable in direct gap semiconductors. An alternative is to incorporate a dopant that can be electrically pumped and has internal gain. Although these are often low gain they can be incorporated in to low-loss devices, in principle, enabling net gain.

The rare earth erbium $\left(\mathrm{Er}^{3+}\right)$ has intrinsic gain and is excited electrically in $\mathrm{Si}$ via band gap recombination. ${ }^{7} \mathrm{Al}-$ though Er has often been mooted as a route to gain in $\mathrm{Si}$, the optical cross sections were supposed too small $\left(\sim 10^{-20} \mathrm{~cm}^{2}\right)$ to achieve sufficient gain, as this would require the incorporation of unrealistic Er concentrations $\left(\sim 10^{20} \mathrm{~cm}^{-3}\right)$. However, if these constraints could be overcome, it would enable the development of fully integrated $\mathrm{Si}$ optical amplifiers and lasers.

We report experimental measurements of optical gain due to $\mathrm{Er}$ in $\mathrm{Si}$. The low gain of the rare earths and the restricted device dimensions of only a few centimeters in semiconductors have previously prevented the measurement

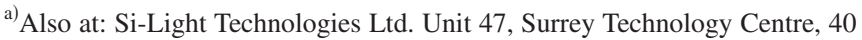
Occam Road, The Surrey Research Park, GU2 7YG, United Kingdom.

${ }^{b)}$ Electronic mail: k.homewood@surrey.ac.uk
}

of the gain coefficients. Here we have developed a technique to enable the measurement of low gain systems in short semiconductor waveguides.

Experiments were carried out on samples fabricated in silicon-on-insulator (SOI) material. The high refractive index $\mathrm{Si}$ overlayer forms a waveguide between the oxide interface and the surface. The sample and the measurement system are shown in Fig. 1. The sample is mounted inside a cryostat. Photoluminescence (PL) is excited within the waveguide at one end of the sample, by the probe laser, set at $488 \mathrm{~nm}$ and modulated at a high frequency. The light passes through a region of the waveguide illuminated by the pump laser and is collected from the far end of the sample. The pump laser, set at $514 \mathrm{~nm}$, is modulated at a much lower frequency and focused to a stripe $250 \mu \mathrm{m}$ wide and $1 \mathrm{~cm}$ long. The output and changes in the output are measured using the technique where two lock-in amplifiers are connected in series. The output intensity is measured as a function of wavelength by the first lock-in amplifier, referenced to the probe frequency. The output of this first lock-in is fed into the second lock-in amplifier. The change, increase or decrease, in the transmitted light is then measured by the second lock-in referenced to the lower modulation frequency. The change in the loss/ gain coefficients due to the optical pumping can be calculated directly from the fractional change in the transmitted

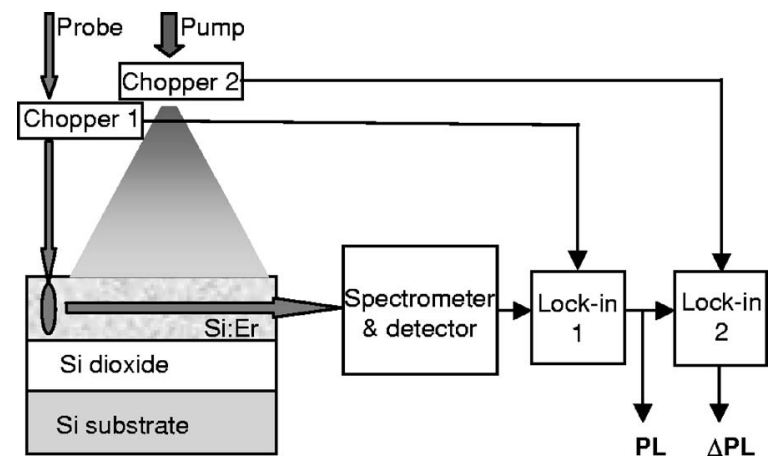

FIG. 1. Schematic of measurement system. Photoluminescence is excited by a probe laser at one end of the sample, modulated at $96 \mathrm{~Hz}$. The PL output, as a function of wavelength, is measured at lock-in 1 referenced to this frequency. The PL passes through the waveguide region optically pumped by the pump laser, modulated at $19 \mathrm{~Hz}$. The change in the probe PL, $\Delta \mathrm{PL}$, is measured by lock-in 2 referenced to the pump modulation frequency. 

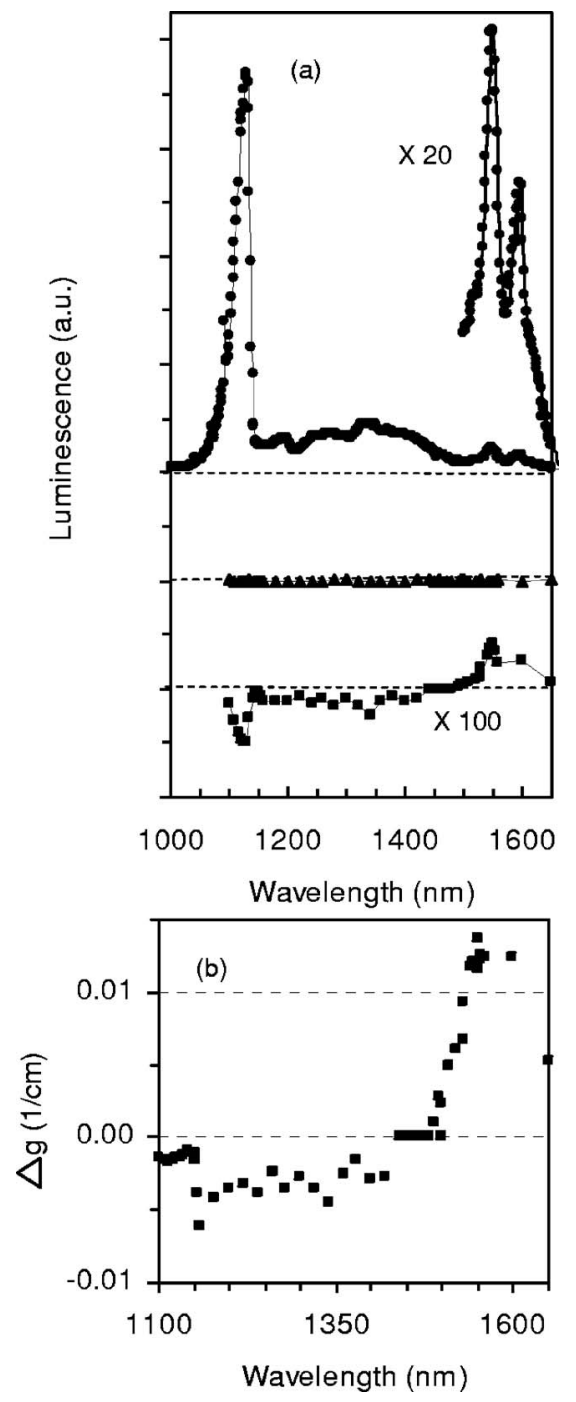

FIG. 2. (a) Curve $1(\bullet)$ shows the PL due to the probe laser only, taken from the output of lock-in 1. The region between 1500 and $1600 \mathrm{~nm}$ has also been expanded vertically to better reveal the structure on the spectrum in this region. Curve $2(\boldsymbol{\Delta})$, coincident with the origin, is the output from lock-in 2 with the pump laser on and probe laser off. Curve 3 (ם) is the change in PL, $\Delta \mathrm{PL}$, with both the probe and pump lasers on and gives the change in the PL due to the probe laser induced by the pump laser. The curves have been displaced for clarity; in all cases the dashed line is the zero axis. (b) Data of (a) converted to $\Delta g$, where $\Delta g=[\ln (1+\Delta \mathrm{PL} / \mathrm{PL})] / L$ with $L$ the pumped length.

light. The main features of this technique are, firstly, the use of internally generated PL from the sample itself as the probe. This avoids the highly problematic, if not impossible, task of efficiently coupling an external broad band light source into a narrow waveguide at low temperatures down a cryostat. More fundamentally, in short lengths of materials with low gain coefficients the effect of the pump is inevitably small (in this case $\sim 0.1 \%-1 \%$ ) and well within the noise level of the probe; consequently, the use of a single lock-in to measure either the effect of the pump on a dc probe or vice versa is insufficient. This limitation is overcome with the suitable, serial connection of two lock-ins described here.

Typical luminescence spectra, measured at $100 \mathrm{~K}$ and with a pump beam power density of $4 \mathrm{~W} \mathrm{~cm}^{-2}$, are shown in Fig. 2(a). Curve $1(\bullet)$ is the PL from the end of the sample due to the probe laser only, measured on lock-in 1. Curve 2 $(\boldsymbol{\Delta})$, coincident with the $x$ axis, is the output from lock-in 2 with only the pump laser on. Clearly no PL due to the pump Downloaded 30 Mar 2009 to 131.227.178.132. Redistribution subje laser is detected showing that lock-in 1 is acting as a perfect

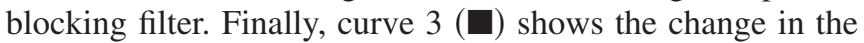
transmitted light from the probe due to the pump laser. Curve 1 is typical of the samples. The peaks at 1130 and $1190 \mathrm{~nm}$ are the phonon assisted band-to-band emission from $\mathrm{Si}$ and the associated phonon replica. ${ }^{1}$ The emission between 1200 and 1500 we believe is associated with residual implant damage. The 1500-1650 region is associated with the Er transitions. The spectral shape for $\mathrm{Er}^{3+}$ over this range is typical of that observed previously in $\mathrm{Si}$ at this temperature ${ }^{8}$ and in particular shows clearly resolved peaks at 1550 and $1595 \mathrm{~nm}$ associated with the two main ${ }^{4} I_{13 / 2}{ }^{4} I_{15 / 2}$ transitions in the $4 f$ shell. It can be seen from the change in PL (curve 3) that the transmitted light has suffered loss at the Si and defect peaks, attributed to the additional absorption due to free carriers generated by the pump laser ${ }^{9}$ but shows net material gain where expected in the Er region. Crucially, this result shows that the gain significantly exceeds any losses due to the additional free carriers needed to achieve it. Figure 2(b) shows the fractional change in transmission converted to change $\Delta g$ in the net material gain where $\Delta g=g^{\prime}-\alpha, g^{\prime}$ is the gain due to the stimulated emission and $\alpha$ is the optical loss. The losses in regions $1000-1500 \mathrm{~nm}$ are clear as is the gain over the $\mathrm{Er}$ region from 1550 to $1650 \mathrm{~nm}$. For this particular experimental run and sample, $\Delta g=0.013 \mathrm{~cm}^{-1}$ for the peak gain and the signal to noise ratio is around $15: 1$, which could be further improved by increasing the averaging time constant on the second lock in. Consequently, we estimate that this technique could detect gain values down to around $0.001 \mathrm{~cm}^{-1}$.

We have measured four SOI samples with different $\mathrm{Si}$ overlayer thicknesses and background doping to allow a greater range of effective concentrations to be accessed. Samples S1, a 2.1- $\mu \mathrm{m}$-thick $\mathrm{Si}$ heavily doped (2 $\left.\times 10^{17} \mathrm{~cm}^{-3}\right) n$-type layer, and $\mathrm{S} 2$, a $2.5 \mu \mathrm{m}$ moderately doped $\left(3 \times 10^{16} \mathrm{~cm}^{-3}\right) p$-type layer, were implanted with $\mathrm{Er}$ at five different energies $(1.5,1.0,0.65,0.4$, and $0.25 \mathrm{MeV})$ and doses $\left(1.6 \times 10^{13}, 1.0 \times 10^{13}, 7.5 \times 10^{12}, 5.0 \times 10^{12}\right.$, and $3.3 \times 10^{12} \mathrm{~cm}^{-2}$, respectively) to provide an essentially uniform doping of $10^{18} \mathrm{~cm}^{-3}$ to a depth of about $0.5 \mu \mathrm{m}$. The total implanted dose was $4.18 \times 10^{13} \mathrm{~cm}^{-2}$, giving an effective concentration, the dose averaged over the waveguide volume of $2.0 \times 10^{17} \mathrm{~cm}^{-3}$ for sample $\mathrm{S} 1$ and of 1.7 $\times 10^{17} \mathrm{~cm}^{-3}$ for sample $\mathrm{S} 2$. These samples were subsequently annealed at $850{ }^{\circ} \mathrm{C}$ for $1 \mathrm{~min}$ in nitrogen ambient. Samples S3 and S4 were phosphorus doped $n$-type Si layers with doping concentrations of $10^{14} \mathrm{~cm}^{-3}$ and 1.5 and $5 \mu \mathrm{m}$ in thickness, respectively. These were identically implanted with $2 \times 10^{13} \mathrm{Er} \mathrm{cm}^{-2}$ at $0.4 \mathrm{MeV}$ and subsequently annealed at $950{ }^{\circ} \mathrm{C}$ for $1 \mathrm{~min}$. This corresponds to a peak concentration of $3 \times 10^{18} \mathrm{~cm}^{-3}$ and an effective concentration of 1.3 $\times 10^{17} \mathrm{~cm}^{-3}$ for sample S3 and $4 \times 10^{16} \mathrm{~cm}^{-3}$ for sample S4. Prior to the Er implant, samples S3 and S4 were also implanted with $10^{15} \mathrm{~B} \mathrm{~cm}^{-2}$ at $30 \mathrm{keV}$ and annealed at $950^{\circ} \mathrm{C}$ for $20 \mathrm{~min}$ in nitrogen ambient to form light emitting diode structures. This process has been previously used to improve and in some situations reverse the temperature quenching of the $\mathrm{Si}$ and Er luminescence in crystalline $\mathrm{Si}$ and provide room temperature operating light emitting diodes. ${ }^{1,8}$

Figure 3(a) shows $\Delta g$, taken at $1550 \mathrm{~nm}$ and normalized to $100 \mathrm{~K}$, as a function of temperature for all the samples. The thermal quenching is significantly reduced in the boron codoped samples and we believe could be completely eliminated in optimized device structures as has been demonto AlP license or copyright; see http://apl.aip.org/apl/copyright.jsp 

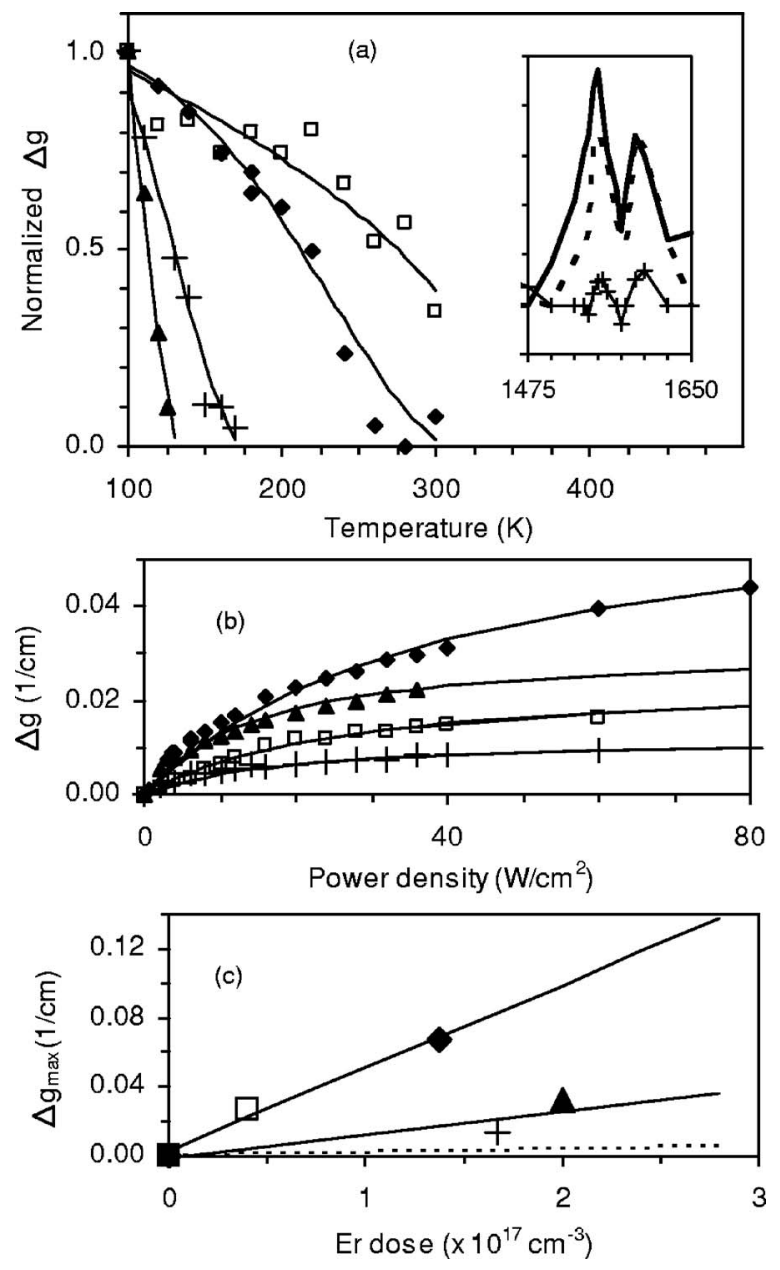

FIG. 3. (a) Normalized $\Delta g$ as a function of temperature, measured at $1550 \mathrm{~nm}$, for samples S1 $(\boldsymbol{\Delta})$, S2 (+), S3 $(\bullet)$, and S4 $(\square)$. The solid lines are guides to the eye. Inset shows spectra of $\Delta g$ for sample S2 for three different temperatures: $80 \mathrm{~K}$ (solid line), $110 \mathrm{~K}$ (dashed line), and $160 \mathrm{~K}$ (crossed line). (b) $\Delta g$ measured at $1550 \mathrm{~nm}$ for the same samples as a function of pump power density. The solid lines are theoretical fits to the data. (c) A plot of $\Delta g_{\max }$, obtained from the theoretical fits to the data in (b) against the effective Er concentration. The dashed line is $g_{\max }^{\prime}$ calculated using the expected value for the optical cross section of $1.8 \times 10^{-20} \mathrm{~cm}^{2}$.

strated for Er electroluminescence in bulk silicon. ${ }^{8}$ Thermal quenching is due to de-excitation of the Er centers back to the $\mathrm{Si}$ and subsequent carrier loss largely due to the strongly temperature dependent nonradiative recombination at the surface. This carrier loss has been previously shown to be reduced by the use of boron doping to produce dislocation loops that when properly engineered can introduce a diffusion barrier decoupling carriers from the nonradiative defects. $^{1}$

The inset of Fig. 3(a) shows $\Delta g$ spectra for sample S2 at different temperatures. The higher resolution used over the gain region has enabled the expected structure of the two main emission lines of the Er gain curve to be resolved. Figure 3(b) shows the power dependence of $\Delta g$. The solid lines are theoretical fits using the standard rate equation model for the $\mathrm{Er}^{3+}$ ion, ${ }^{10}$ using only two fitting parameters.
Terms for cooperative up-conversion and excited state absorption were not included, indicating that these loss mechanisms are negligible in the Er-doped Si system. ${ }^{10,11}$ Values of the maximum change in net gain, $\Delta g_{\max }$, for the differently doped samples are obtained by extrapolation of the fits of $\Delta g$ to the saturation region and are $0.032 \pm 0.001$, $0.0128 \pm 0.0003,0.0673 \pm 0.002$, and $0.0267 \pm 0.0007 \mathrm{~cm}^{-1}$ for samples S1, S2, S3, and S4, respectively. Figure 3(c) shows $\Delta g_{\max }$ as a function of the effective Er concentration. For comparison we have also plotted the maximum gain, $g_{\max }^{\prime}\left(g_{\max }^{\prime}=\sigma N\right.$, where $\sigma$ is the optical emission cross section and $N$ is the effective Er concentration), using the expected value of $1.8 \times 10^{-20} \mathrm{~cm}^{2}$ for the optical emission cross section. ${ }^{7}$ Measured $\Delta g_{\max }$ values are around six times higher in the Er-doped samples and around 30 times higher for the Er samples codoped with boron than expected. This gives an effective lower limit to the optical cross section for the Er in the boron doped samples of $(5.0 \pm 0.1) \times 10^{-19} \mathrm{~cm}^{2}$. The lower $\Delta g_{\max }$ measurements in the Er only samples is attributed to the faster temperature quenching than in the samples codoped with boron. The mechanism for the increase in the stimulated emission cross section in $\mathrm{Si}$ over that seen in glassy hosts is currently unclear. However, a comparable enhancement of gain has been previously observed in $\mathrm{Er}$ in $\mathrm{SiO}_{2}$ when $\mathrm{Si}$ nanocrystals (nc-Si), which mediate the excitation, have been introduced. ${ }^{10}$ In this work they obtained a value for the emission cross section of $(2.0 \pm 0.5)$ $\times 10^{-19} \mathrm{~cm}^{2}$ for their Er-doped nc-Si sensitized $\mathrm{SiO}_{2}$-half the value obtained here on Er-doped bulk Si. At an Er concentration $10^{19} \mathrm{~cm}^{-3}$ we would expect to obtain a gain of $22 \mathrm{~dB} \mathrm{~cm}^{-1}$.

In summary we have demonstrated and measured optical gain in Er-doped Si. The values measured far exceed previous expectations of the likely gain values in this system. The gain values obtained mean that the development of optical amplifiers and laser devices using this approach now becomes a realistic possibility at achievable, optically active doping levels. This system therefore offers a potential route to fully CMOS compatible, all silicon, photonic integrated circuits.

${ }^{1}$ W. L. Ng, M. A. Lourenço, R. M. Gwilliam, S. Ledain, G. Shao, and K. P. Homewood, Nature (London) 410, 192 (2001).

${ }^{2}$ O. Boyraz and B. Jalali, Opt. Express 12, 5269 (2004).

${ }^{3}$ H. S. Rong, A. S. Liu, R. Jones, O. Cohen, D. Hak, R. Nicolaescu, A. Fang, and M. Paniccia, Nature (London) 433, 292 (2005).

${ }^{4}$ H. S. Rong, R. Jones, A. S. Liu, O. Cohen, D. Hak, A. Fang, and M. Panicia, Nature (London) 433, 725 (2005).

${ }^{5}$ M. A. Foster, A. C. Turner, J. E. Sharping, B. S. Schmidt, M. Lipson, and A. L. Gaeta, Nature (London) 441, 960 (2006).

${ }^{6}$ S. G. Cloutier, P. A. Kossyrev, and J. Xu, Nat. Mater. 4, 887 (2005).

${ }^{7}$ N. Hamelin, P. G. Kik, J. F. Suyver, K. Kikoin, A. Polman, A. Schonecker, and F. W. Saris, J. Appl. Phys. 88, 5381 (2000).

${ }^{8}$ M. A. Lourenço, M. Milosavljevic, S. Galata, M. S. A. Siddiqui, G. Shao, R. M. Gwilliam, and K. P. Homewood, Vacuum 78, 551 (2005).

${ }^{9}$ L. Pavesi, Mater. Today 1, 18 (2005).

${ }^{10}$ H. S. Han, S. Y. Seo, J. H. Shin, and N. Park, Appl. Phys. Lett. 81, 3720 (2002).

${ }^{11}$ G. N. vandenHoven, R. J. I. M. Koper, A. Polman, C. vanDam, J. W. M. vanUffelen, and M. K. Smit, Appl. Phys. Lett. 68, 1886 (1996). 